\title{
Discovery of 9-phenylacridinediones as highly selective butyrylcholinesterase inhibitors through structure-based virtual screening
}

\author{
Jacques Joubert*, Erika Kapp \\ Pharmaceutical Chemistry, School of Pharmacy, University of the Western Cape, Private Bag X17, Bellville 7535, South Africa
}

\section{A R T I C L E I N F O}

\section{Keywords:}

Alzheimer's disease

Butyrylcholinesterase inhibitors

Virtual screening

Docking

9-Phenylacridinediones

\begin{abstract}
A B S T R A C T
Butyrylcholinesterase (BuChE) is considered a promising drug target as it plays an important role in the progression of late stage Alzheimer's disease (AD). Two compound libraries were selected and 64124 amine containing moieties were screened using a hierarchical virtual screening protocol to discover new selective BuChE inhibitors. From these and subsequent docking experiments, 9-phenylacridinedione (9-PAD) was identified as a promising scaffold for selective inhibition of BuChE. Selected top dock scored 9-PADs were assayed and compounds 3 and 6 exhibited potent and highly selective human BuChE inhibition ( $\mathrm{IC}_{50}$ : $98 \mathrm{nM}$ and $142 \mathrm{nM}$, respectively). Both molecules were also predicted to show sufficient brain permeability, not have any substantial toxicities, especially hepatotoxicity, and no significant in vitro cytotoxicity against SH-SY5Y neuroblastoma cells at concentrations up to $100 \mu \mathrm{M}$. These findings indicate that 9-PAD is a promising lead structure for the development of agents able to treat late stage AD.
\end{abstract}

Alzheimer's disease (AD) is a devastating neurodegenerative disorder characterized by a progressive and steady deterioration in mental capacity, combined with negative behavioural and psychiatric symptoms. $^{1,2}$ Throughout the world it is estimated that 50 million people are affected by dementia, with most cases linked to AD. This number is predicted to increase 3 -fold within the following 30 years. ${ }^{3}$ Therefore, new and improved therapeutic agents are of the utmost importance in order to curb this devastating disorder. The pathophysiology of AD is complex and multifactorial, and researchers have proposed a variety of mechanisms which contribute to the disease state. ${ }^{4}$ These include cholinergic dysfunction, ${ }^{5,6}$ deposition of amyloid- $\beta$ plaques, ${ }^{7}$ accumulation of tau-proteins ${ }^{6,7}$ and oxidative stress. ${ }^{8}$

Current treatment options are mainly focussed on the cholinergic hypotheses which states that degradation of acetylcholine (ACh) containing neurons and subsequent shortage of the neurotransmitter ACh contributes substantially to the cognitive decline associated with AD. ${ }^{5,6,9}$ Hydrolysis of ACh in the CNS is mainly regulated by two cholinesterase enzymes namely; acetylcholinesterase (AChE) and butyrylcholinesterase (BuChE). ${ }^{10} \mathrm{AChE}$ is accountable for $80 \%$ of the $\mathrm{ACh}$ activity and is almost 10000 times more active at hydrolysing ACh than BuChE under normal conditions. ${ }^{11,12}$ In addition, AChE is mainly found in neurons and BuChE is generally expressed in glial- and endothelial cells in the brain. ${ }^{13,14}$ Therefore, the use of AChE inhibitors such as galanthamine, ${ }^{15}$ rivastigmine ${ }^{16}$ and donepezil ${ }^{17,18}$ have been the main therapeutic approach used to increase ACh levels in neurons of patients suffering from AD. However, studies have found that during the progression of $\mathrm{AD}$ the levels of $\mathrm{AChE}$ show a steady decrease, which in turn leads to BuChE taking over the hydrolysis of ACh. Under these conditions BuChE may increase by up to $165 \%$ of its usual physiological level. ${ }^{19,20}$ Thus, BuChE and the subsequent inhibition thereof is a suggested therapeutic target that may be beneficial for the treatment of advanced stage AD. Furthermore, the development of highly selective BuChE inhibitors may avoid some of the common cholinergic side effects associated with AChE inhibition. ${ }^{21}$ In recent years several selective BuChE have been developed, including diarylinidazole-, ${ }^{22}$ cymserine-- ${ }^{23}$ benzofuran- ${ }^{24}$ and isosorbide-based ${ }^{25}$ compounds. However, none of these compounds have made it further than in vitro studies, and the need for the discovery of new selective BuChE inhibitors that may make it to a drug candidate stage therefore still remains.

In order to develop highly selective inhibitors of the human BuChE (huBuChE) enzyme, it is essential to study the differences in the protein structures between human AChE (huAChE) and huBuChE, especially within the active sites of the enzymes. The homology of huAChE and huBuChE indicates that the two enzymes share $65 \%$ identical amino acid arrangements with a similar general structure. ${ }^{26-28}$ The active sites of the two enzymes are comprised of a catalytic anionic site and choline binding pocket which are situated at the bottom of a $\sim 20 \AA$ deep gorge. The main difference between these two enzymes are the presence and

\footnotetext{
* Corresponding author. Tel.: +27 219592195.

E-mail address: jjoubert@uwc.ac.za (J. Joubert).
} 

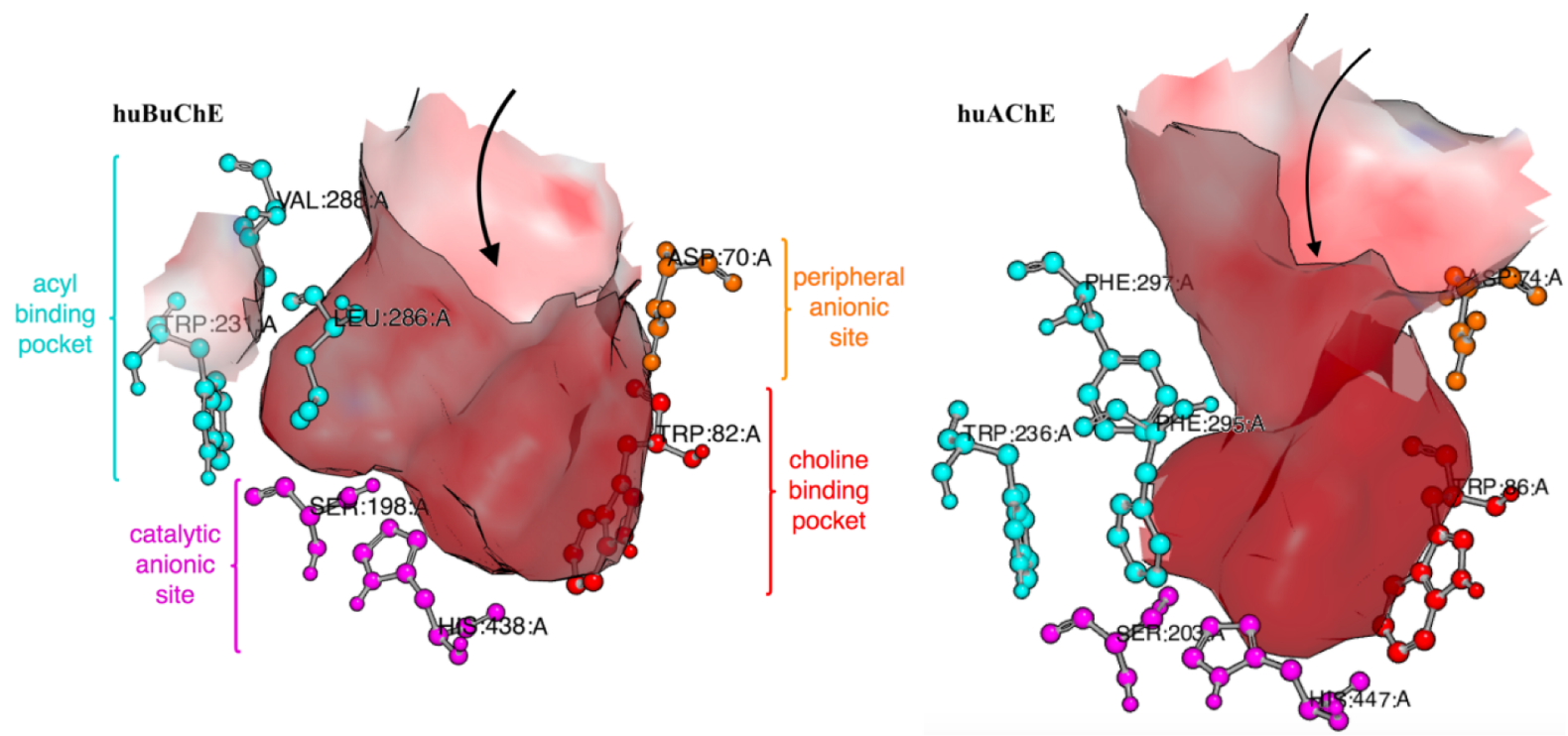

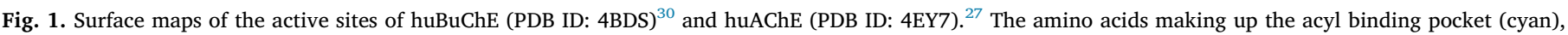

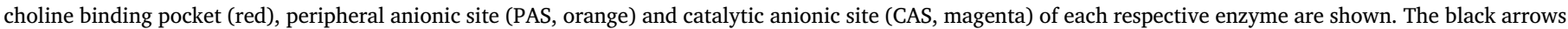
indicate the entry direction of ligands and substrates.

extent of sub-regions within the gorge which includes an acyl-binding pocket and a peripheral anionic site (Fig. 1). The differences observed in the acyl-binding site are especially important. In huAChE this site accommodates two aromatic amino acids Phe295 and Phe297 which are respectively replaced by aliphatic amino acids Leu286 and Val288 in huBuChE. ${ }^{26-28}$ Due to the presence of the two bulky phenylalanine residues in the acyl binding pocket, binding and hydrolysis of larger compounds are restricted in huAChE because of the smaller ligand binding cavity volume. ${ }^{29}$ In huBuChE, replacement of these residues with the two flexible smaller aliphatic amino acids allows this site to accommodate larger compounds. This structural difference between the active sites of these enzymes may therefore be strategically used in order to identify highly selective inhibitors of the huBuChE enzyme.

In the present study, a structure-based virtual docking methodology was applied in an attempt to identify novel highly selective huBuChE inhibitors (Fig. 2, see Supplementary File S1 for experimental details). To reach this objective, we screened a combination of the Maybridge screening collection (https://www.maybridge.com) and Aldrich ${ }^{\mathrm{CPR}}$ (https://www.sigmaaldrich.com) compound databases. These databases were selected because they contain structurally diverse purchasable compounds, especially the Maybridge collection. ${ }^{31}$ In addition, most of the compounds in these databases obey Lipinski's "rule of five" 32 and also demonstrate good predicted ADME profiles, which make them ideal candidates to identify drug-like compounds using structure-based virtual screening experiments. Around 265000 druglike compounds were compiled using these databases. As described in the literature, the majority of known cholinesterase ligands contain basic or permanently charged nitrogens. This is because acidic protein residues lining both the CAS (Glu197 in huBuChE) and PAS (Asp70 in huBuChE) are able to attract positively charged ligands. ${ }^{33,34}$ In addition, studies have indicated that amine containing compounds are able to interact with the CAS through cation- $\pi$ interactions. ${ }^{35,36}$ Therefore, the compiled database was narrowed down using OGREP, a commandline tool of OpenBabel 2.2.3, ${ }^{37}$ to a focused database of 64124 compounds bearing an amine moiety.

The initial virtual screen was performed using FRED 2.2.5 docking software (OpenEye Scientific Software, Inc., Santa Fe, NM, USA; www.eyesopen.com) and the huBuChE protein structure in complex with tacrine (PDB: 4BDS). ${ }^{30}$ The amine moiety containing database was docked into the active site cavity of huBuChE. The top pose of each docked compound where at least one heavy atom was within close proximity to Trp82 and one with Ser198 was then ranked according to their respective Chemgausse 3 scores. ${ }^{38-40}$ These custom constraints were selected based on the known binding modes of inhibitors (e.g. tacrine - PDB ID: 4BDS; decamethonium - PDB ID: 6EP4; ethopropazine - PDB ID: 6EQP; propidium - PDB ID: 6ESJ and huperzine A - PDB ID: 1VOT) and substrates (e.g. choline, butyrate and glycerol - PDB IDs: $1 \mathrm{P} 01$ and 1P0M) within the active site of the enzyme. ${ }^{26-29}$ Results from this initial screen indicated that 8 out of the top 40 scored compounds consisted out of a 9-phenylacridinedione (9-PAD) scaffold or closely related derivative thereof (see Supplementary File S2). To the best of our knowledge 9-PADs have not yet been described in the literature as ChE inhibitors. In addition, a number of structurally- and/or shape similar compounds compared to the 9-PADs, such as dihydropyridineand phenothiazine derivatives, were also observed within the top 40 docked compounds. Both dihydropyridine- and phenothiazine derivatives have been described in the literature as having ChE inhibitory abilities. ${ }^{41-44}$ This prompted our interest to explore 9-PAD as a potential novel scaffold able to inhibit huBuChE. In addition, 9-PAD has some structural features similar to the known non-selective ChE inhibitor, tacrine, ${ }^{45}$ which further justified additional investigation (Fig. 3).

In the next step, the ZINC drug-like compound database containing around 11.3 million purchasable compounds was downloaded. ${ }^{46}$ OGREP was used to extract all compounds from the ZINC database containing the 9-PAD scaffold. ${ }^{37} \mathrm{~A}$ total of 1374 9-PADs were identified. These 9-PADs were also docked using FRED 2.2.5 with huBuChE (PDB: 4BDS) ${ }^{30}$ in the same manner as described for the initial screen. The compounds were again ranked according to their Chemgauss3 score and the top 40 scored 9-PADs were selected for further investigation (see Supplementary File S3). In order to predict the selectivity of the top 40 9-PADs for huBuChE over huAChE a further docking experiment was done where the huAChE enzyme in complex with donepezil (PDB: 4EY7) ${ }^{27}$ was used. FRED 2.2.5 docking software predicted that none of the top 40 9-PADs would have the ability to optimally bind to the huAChE active site, as none of the predicted conformations were within the proximity of the custom constrained amino acids Trp86 and Ser203. ${ }^{26-29}$ This may be due to the structural bulkiness and/or orientation of the 9-PADs leading to their inability to effectively pass through the narrow gorge and/or fit within the smaller, compared to BuChE, active site of huAChE.

To confirm the promising results obtained from the docking experiments, seven compounds (2-8) ranging within the top 40 scored 9 - 


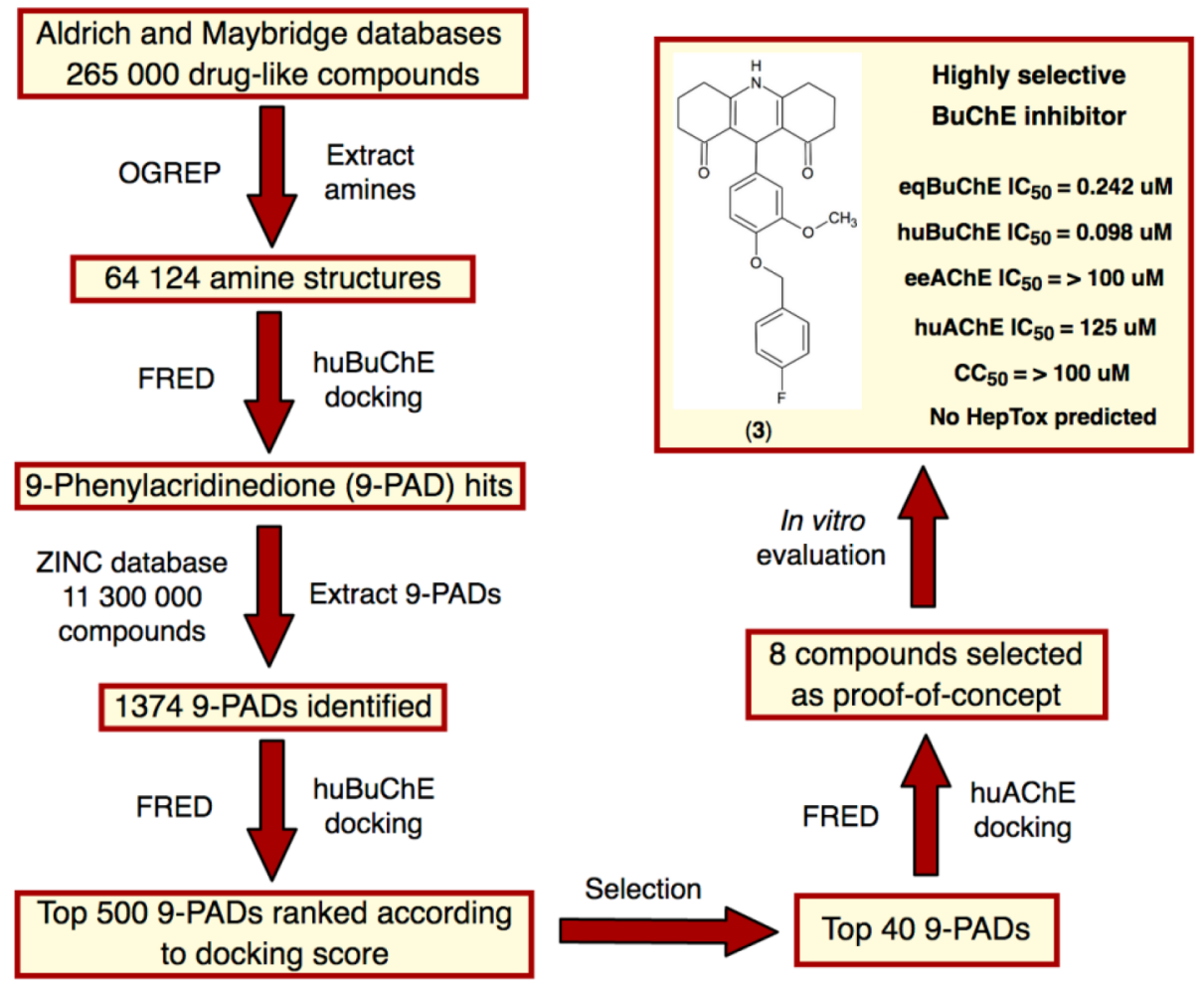

Fig. 2. Flow scheme showcasing the hierarchical structure-based virtual screening protocol.

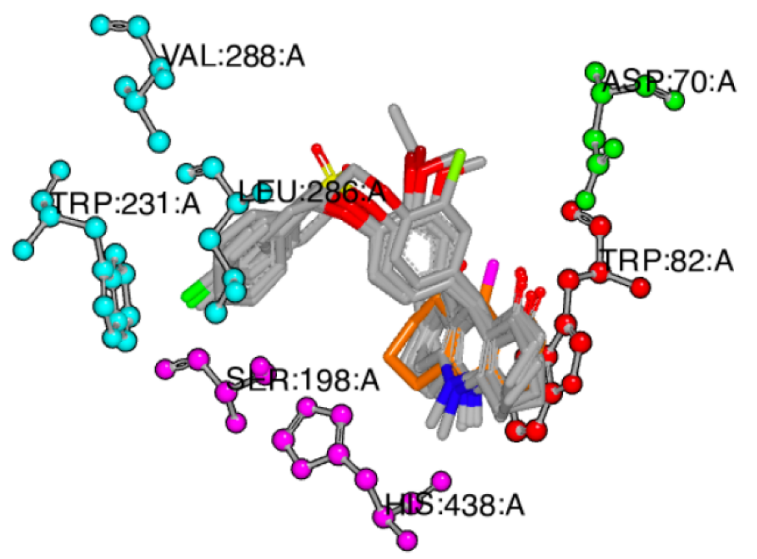

Fig. 3. Binding orientation of all selected 9-PADs (1-8, shown in gray) docked into the active site cavity of huBuChE. Tacrine is shown in orange.

PADs were selected for further evaluation as initial proof-of-concept (Table 1). Compound 1, an unsubstituted 9-PAD derivative, was not scored within the top 500 9-PAD's but was included as a reference to explore the docking accuracy and ability to predict agents with potent huBuChE inhibitory activities. The selection of these compounds was also based on availability, ability to be shipped to South Africa and cost.

The binding interactions, conformation and orientation of the purchased 9-PADs (1-8) within the huBuChE active site were investigated (Table 1, Figs. 3-5). Interactions between the individual 9-PADs and Trp82, ${ }^{26,47}$ Trp231, ${ }^{48}$ Ser198 and His438 ${ }^{49,50}$ were considered significant as these are important for inhibitory activity. Additionally, the ability of these 9-PADs to access the acyl site is also substantially important for potential selectivity towards huBuChE as this pocket is larger than in huAChE (Fig. 1). ${ }^{29}$

All the docked 9-PADs showed promising binding modes with the acridinedione moiety binding in the same vicinity as the co-complexed ligand, tacrine, ${ }^{30}$ and the methylbenzene moiety occupying the acyl- binding pocket (Fig. 3). The phenyl moiety directly connected to the acridinedione is orientated towards Asp70. In addition, the halogen or methoxy substitutions on this phenyl group presides around the backbone atoms of Asp70 in the PAS, thus providing stability to the 9-PADs at the entrance of the active site gorge. This observation is important as the majority of the 9-PADs scored within the top-40 compounds (see Supplementary File S2) contained substitutions at this position which likely contributes to enhanced binding stability.

As for the binding interactions, compounds 1-3, 5 and 6 were able to from a $\mathrm{H}-\pi$ interaction between Trp 82 and the acridinedione moiety, while the methylbenzene group forms $a \pi-\pi$ interaction with Trp231. The unsubstituted amine of the acridinedione was able to interact with His438 through a hydrogen bond interaction (Table 1, Figs. 4 and 5). The two compounds within this series containing a methyl substitution on the amine moiety ( 4 and 7), lacked this interaction with His438. This contributed to a slight shift in their binding orientation leading to a loss of interactions with Trp82 and Trp231. Compound 8, containing the sulfonate moiety also exhibited a lack of predicted binding interactions with these tryptophan residues, but maintained an interaction with His438. However, compounds 4, 7 and 8 still showed convenientbinding modes within the huBuChE active site and should still exhibit moderate huBuChE inhibitory activity based on these docking results (Table 1, Figs. 4 and 5). It was noted that compounds 1-8 did not directly interact with the catalytic residue Ser198, which has been reported as an important amino acid, amongst others, for cholinesterase inhibition. ${ }^{26-29}$ However, the cyclohexanone group on the one side of the acridinedione moiety in these 9-PADs points toward Ser198 (average distance between closest non-hydrogen atoms is $3.5 \AA$ ). This therefore offers an opportunity for substitutions on this position of the cyclohexanone group to potentially improve the potency of these compounds.

In an attempt to further investigate the results from the initial huAChE screen, the docking experiment was repeated for compounds 1-8. However, in this case the custom constraints were removed in order for the binding modes to be generated. The results from the top scored poses, indicated that the acridinedione moiety is orientated 
Table 1

ZINC ID, supplier, structures, dock scores and huBuChE active site binding interactions of 9-PADs 1-8 and tacrine.

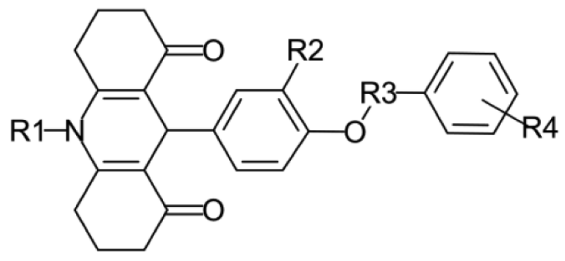

\begin{tabular}{|c|c|c|c|c|c|c|c|c|}
\hline Compound & ZINC ID & Supplier & R1 & $\mathrm{R} 2$ & R3 & R4 & Chemgausse3 Score & Interactions \\
\hline 1 & 627289 & Sigma Aldrich & $-\mathrm{H}$ & $-\mathrm{H}$ & $-\mathrm{CH}_{2-}$ & $-\mathrm{H}$ & -12.24 & Trp82, His438, Trp231 \\
\hline 2 & 00689050 & Molport & $-\mathrm{H}$ & $-\mathrm{OCH}_{2} \mathrm{CH}_{3}$ & $-\mathrm{CH}_{2-}^{-}$ & $-\mathrm{H}$ & -17.68 & Trp82, His438, Trp231 \\
\hline 3 & 00796178 & Molport & $-\mathrm{H}$ & $-\mathrm{OCH}_{3}$ & $-\mathrm{CH}_{2-}$ & p-fluoro & -17.36 & Trp82, His438, Trp231 \\
\hline 4 & 01158419 & Molport & $-\mathrm{CH}_{3}$ & $-\mathrm{OCH}_{3}$ & $-\mathrm{CH}_{2-}$ & p-fluoro & -17.04 & Glu197 \\
\hline 5 & 20109989 & Molport & $-\mathrm{H}$ & $-\mathrm{Br}$ & $-\mathrm{CH}_{2-}$ & $-\mathrm{H}$ & -17.43 & Trp82, His438, Trp231 \\
\hline 6 & 1012743 & Molport & $-\mathrm{H}$ & $-\mathrm{Br}$ & $-\mathrm{CH}_{2-}^{-}$ & m-fluoro & -17.82 & Trp82, His438; Gly116, Trp231 \\
\hline 7 & 01156953 & Molport & $-\mathrm{CH}_{3}$ & $-\mathrm{Cl}$ & $-\mathrm{CH}_{2-}$ & p-chloro & -17.24 & Glu197 \\
\hline 8 & 20109979 & Molport & $-\mathrm{H}$ & $-\mathrm{O}-\mathrm{CH}_{3}$ & $-\mathrm{SO}_{2-}^{-}$ & $\mathrm{p}-\mathrm{CH}_{3}$ & -17.20 & Glu197, His438, Gly117 \\
\hline Tacrine & 19014866 & Sigma Aldrich & - & - & - & - & n.d. & $\operatorname{Trp} 82$ \\
\hline
\end{tabular}

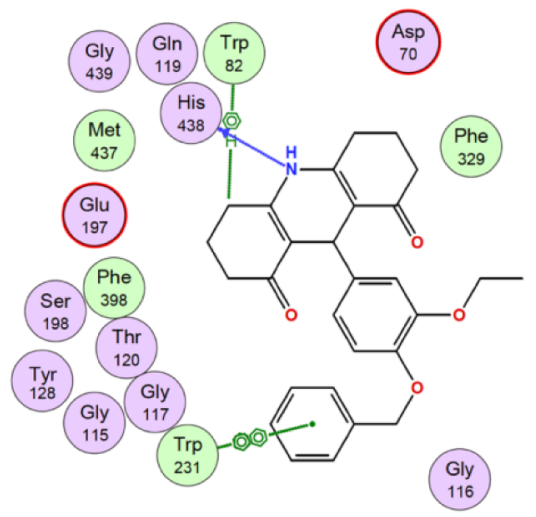

2

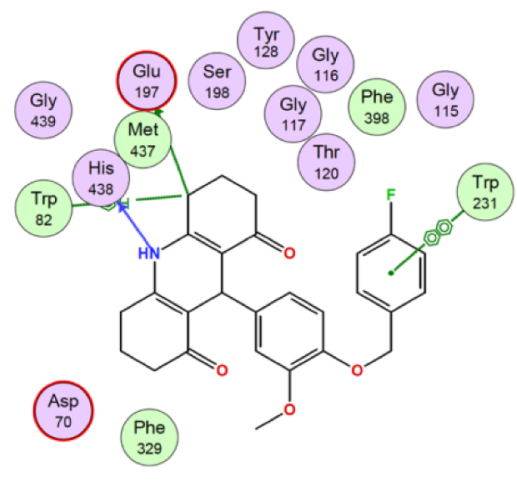

3

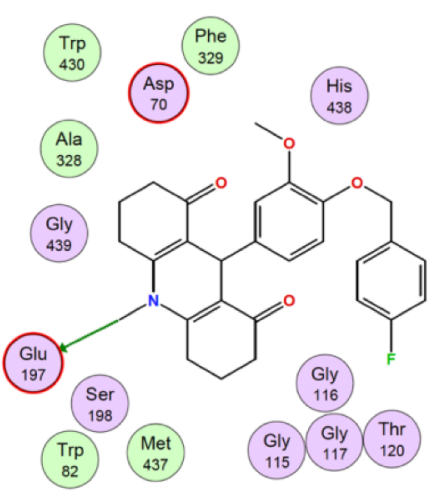

4

Fig. 4. Interaction maps of compounds 2,3 and 4 within the huBuChE active site.

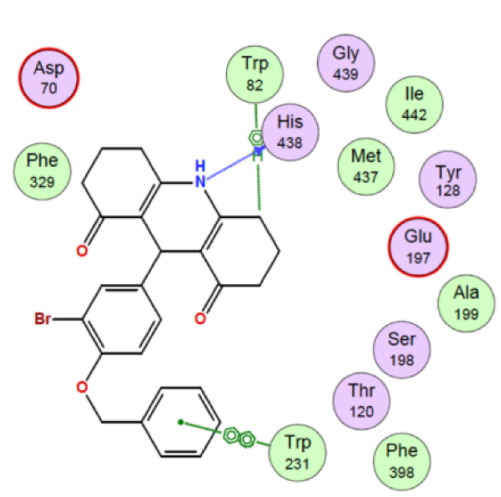

5

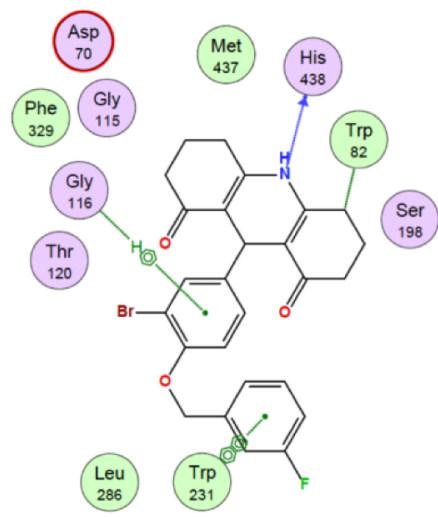

6

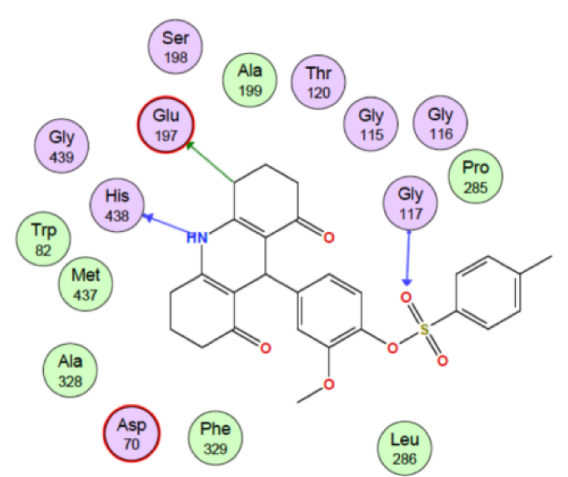

8

Fig. 5. Interaction maps of compounds 5, 6 and 8 within the huBuChE active site.

towards the PAS region within the proximity of the PAS amino acid Asp74 (Fig. 6). This observation indicates that the acridine moiety within these 9-PADs does not have the ability to effectively pass through the narrow gorge separating the PAS and the CAS. Furthermore, tacrine is known to bind in close proximity to Trp 86 and form a $\pi-\pi$ interaction with this residue in AChE. ${ }^{51}$ In the case of these 9-PADs none were predicted to bind in this manner (Fig. 6). In addition, the 9-
PADs were unable to interact with the acyl binding pocket as was observed in huBuChE, because of their structural bulkiness and less than optimal orientation. Therefore, these 9-PADs are not expected to show any significant huAChE inhibitory activity based on these docking results.

The eight compounds selected from the virtual screening results were initially evaluated in vitro for their inhibition on equine BuChE 


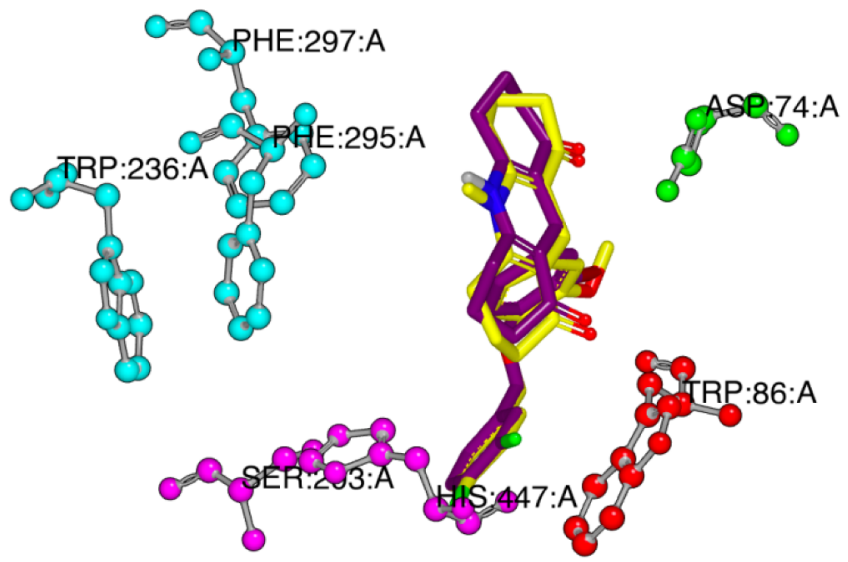

Fig. 6. Binding orientation of representative 9-PADs 4 (purple) and 6 (yellow) docked in the active site cavity of huAChE.

Table 2

In vitro $\mathrm{IC}_{50}$ values $(\mu \mathrm{M} \pm \mathrm{SEM}, \mathrm{n}=3$ ) of test compounds for eqBuChE and eeAChE.

\begin{tabular}{llcl}
\hline Compound & eqBuChE & eeAChE $(\%)^{\mathrm{a}}$ & SI BuChE $^{\mathrm{b}}$ \\
\hline $\mathbf{1}$ & $6.010 \pm 0.42$ & $>100(24)$ & $>17$ \\
$\mathbf{2}$ & $0.752 \pm 0.04$ & $>100(19)$ & $>133$ \\
$\mathbf{3}$ & $0.242 \pm 0.03$ & $>100(27)$ & $>412$ \\
$\mathbf{4}$ & $2.440 \pm 0.21$ & $>100(33)$ & $>41$ \\
$\mathbf{5}$ & $0.633 \pm 0.05$ & $>100(14)$ & $>158$ \\
$\mathbf{6}$ & $0.388 \pm 0.08$ & $>100(22)$ & $>258$ \\
$\mathbf{7}$ & $1.658 \pm 0.12$ & $>100(23)$ & $>60$ \\
$\mathbf{8}$ & $0.959 \pm 0.09$ & $>100(15)$ & $>104$ \\
Tacrine & $0.110 \pm 0.01$ & $0.140 \pm 0.03$ & $>1.27$
\end{tabular}

${ }^{\mathrm{a}}$ Percentage inhibition at $100 \mu \mathrm{M}$ is indicated in brackets. ${ }^{\mathrm{b}}$ eqBuChE selectivity index $=\mathrm{IC}_{50}$ (eeAChE)/IC $\mathrm{I}_{50}$ (eqBuChE). The selectivity was calculated at $100 \mu \mathrm{M}$ on eeAChE because of solubility problems at higher concentrations.

(eqBuChE) and electric eel AChE (eeAChE), using a modified method of Ellman, ${ }^{52}$ as previously described by our group. ${ }^{53-55}$ Inhibitory activities were observed for all the compounds with $\mathrm{IC}_{50}$ values ranging between 0.242 and $6.01 \mu \mathrm{M}$ (Table 2). What was interesting was that compound 1 showed significantly less potent inhibition (between 3 and 28 -fold) compared to the other evaluated 9-PADs. This is in line with the docking experiments as 1 was not predicted within the top 5009 PADs. Therefore, this indicates that substitutions on the two phenyl groups of these 9-PADs leads to a marked increase in activity. In addition, compounds 4 and 7 containing the $-\mathrm{N}^{-\mathrm{CH}_{3}}$ group was significantly less potent than their secondary amine structural counterparts $(\mathbf{1}-\mathbf{3}, \mathbf{5}, \mathbf{6})$. This may be ascribed to the lack of important binding interactions (i.e. with Trp82, His438 and Trp231, Fig. 4) as described in the docking results (Table 1 ). The test compounds were further assayed against eeAChE and the results indicated a high degree of selectivity for eqBuChE for all compounds (Table 2, SI: 17 to 412). These inhibition results revealed that $\mathbf{3}$ and $\mathbf{6}$ are the most potent and selective eqBuChE inhibitors, with $\mathrm{IC}_{50}$ values of $0.243 \mu \mathrm{M}$ and $0.388 \mu \mathrm{M}$, respectively.

Because of the promising inhibition and selectivity of $\mathbf{3}$ and $\mathbf{6}$ for eqBuChE, our next step was to evaluate their inhibitory potential on huBuChE and huAChE in vitro. Compared to eqBuChE, both 3 and 6 were around 2.5-times more active at inhibiting huBuChE, and showed very limited huAChE inhibition (Table 3). Therefore, both $\mathbf{3}$ and $\mathbf{6}$ can be considered as highly selective sub-micromolar inhibitors of huBuChE. Furthermore, the inherent toxicity of $\mathbf{3}$ and $\mathbf{6}$ toward SH-SY5Y cells was investigated utilizing a modified MTT assay described by our group. ${ }^{54-56}$ The viability of the cells was not compromised by the test compounds up to concentrations of $100 \mu \mathrm{M}$ (Table 3). The MTT is a cell proliferation assay, and these results indicates that there were low levels of inhibition of cell proliferation, which would suggest the compounds to be largely devoid of cytotoxicity. In addition, the compounds were screened for potential toxicities on a number of toxicity targets and blood-brain barrier (BBB) permeation using in silico models (see Supplementary File S1, Table S1). Both compounds were predicted to show limited toxicities and adequate ability to cross the BBB (Table 3 ). It is also promising that none of the 9-PADs were predicted to show hepatotoxicity, as is the case for tacrine that lead to its subsequent removal from the pharmaceutical market (Supplementary File S1).

In conclusion, a structure-based virtual docking methodology was employed in an attempt to discover novel highly selective huBuChE inhibitors. From these and subsequent docking experiments, 9-phenylacridinedione (9-PAD) was identified as a promising scaffold for selective inhibition of the huBuChE enzyme. 9-PADs 3 and 6 demonstrated the strongest inhibitory activities and were further tested against huBuChE and huAChE. Both 3 and 6 showed a 2.5-fold increase in huBuChE activity ( $\mathrm{IC}_{50}$ : $98 \mathrm{nM}$ and $142 \mathrm{nM}$, respectively) compared to eqBuChE. Only limited huAChE inhibition was observed, with 3 showing a 1275-fold selectivity for huBuChE. Both molecules were also not predicted have any significant toxicities, especially hepatotoxicity, or in vitro cytotoxicity against SH-SY5Y cells at concentrations up to $100 \mu \mathrm{M}$. The significant and highly selective BuChE inhibitory potencies of these 9-PADs indicate that they could be used as promising lead compounds for the development of new agents for the treatment of late stage $\mathrm{AD}$. It should also be noted that there are 1374 purchasable 9PADs available on the ZINC compound database ${ }^{46}$ and a larger biological screening endeavour may identify available 9-PADs with even greater therapeutic potential.

\section{Declaration of Competing Interest}

The authors declare that they have no known competing financial interests or personal relationships that could have appeared to influence the work reported in this paper.

Table 3

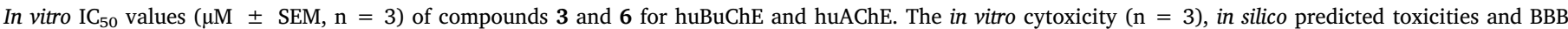
penetration are also included.

\begin{tabular}{|c|c|c|c|c|c|c|}
\hline Compound & huBuChE & huAChE & SI huBuChE ${ }^{\mathrm{b}}$ & $\%$ Cytotoxicity at $100 \mu \mathrm{M}$ & Predicted toxicities ${ }^{\mathrm{d}}$ & BBB penetration ${ }^{\mathrm{e}}$ \\
\hline 3 & $0.098 \pm 0.02$ & $125 \pm 4.52$ & 1275 & $14 \pm 1.22$ & none & Yes $(94 \%)$ \\
\hline 6 & $0.142 \pm 0.05$ & $>100(39)^{\mathrm{a}}$ & $>704^{c}$ & $18 \pm 2.03$ & none & Yes $(97 \%)$ \\
\hline Tacrine & $0.044 \pm 0.01$ & $0.100 \pm 0.05$ & 2.27 & n.d. & $\begin{array}{l}\text { Hepatotoxicity } \\
\text { Mutagenicity }\end{array}$ & Yes $(99 \%)$ \\
\hline
\end{tabular}

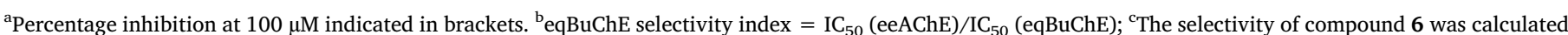

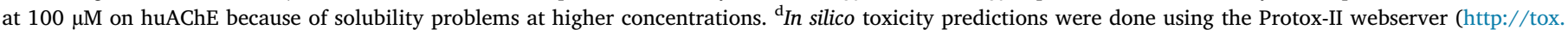

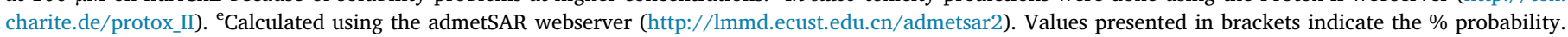
n.d. $=$ not determined. 


\section{Acknowledgements}

We thank OpenEye Scientific Software, Santa Fe, NM, USA (www.eyesopen.com) for free academic licenses of their software and the National Research Foundation of South Africa for financial support.

\section{Appendix A. Supplementary data}

Supplementary data to this article can be found online at https:// doi.org/10.1016/j.bmcl.2020.127075.

\section{References}

1. Burns A, Jacoby R, Levy R. Behavioral abnormalities and psychiatric symptoms in Alzheimer's disease: preliminary findings. Int Psychogeriatr. 1990;2:25-36.

2. Tarawneh R, Holtzman DM. The clinical problem of symptomatic Alzheimer disease and mild cognitive impairment. Cold Spring Harb Perspect Med. 2012;2:a006148.

3. Alzheimer's Disease International. Alzheimer's disease International. World Alzheimer Report 2018. The state of the art of dementia research: new frontiers. London: Alzheimer's Disease International; 2018.

4. Moya-Alvarado G, Gershoni-Emek N, Perlson E, Bronfman FC. Neurodegeneration and Alzheimer's disease (AD). What can proteomics tell us about the Alzheimer's brain? Mol Cell Proteomics. 2016;15:409-425.

5. Bartus RT, Dean RL, Beer B, Lippa AS. The cholinergic hypothesis of geriatric memory dysfunction. Science. 1982;217:408-414.

6. Scarpini E, Schelterns P, Feldman H. Treatment of Alzheimer's disease; current status and new perspectives. Lancet Neurol. 2003;2:539-547.

7. Sinha S, Lieberburg I. Cellular mechanisms of B-amyloid production and secretion. Proc Natl Acad Sci USA. 1999;96:11049-11053.

8. Gella A, Durany N. Oxidative stress in Alzheimer disease. Cell Adhes Migr 2009;3:88-93.

9. Ferreira-Vieira TH, Guimaraes IM, Silva FR, Ribeiro FM. Alzheimer's disease: targeting the cholinergic system. Curr Neuropharmacol. 2016;14:101-115.

10. Borda C. Alzheimer's disease and memory drugs. New York: Infobase Publishing; 2006:45-56

11. Perry E, Walker M, Grace J, Perry R. Acetylcholine in mind: a neurotransmitter correlate of consciousness? Trends Neurosci. 1999;22:273-280.

12. Bazelyansky M, Robey E, Kirsch JF. Fractional diffusion-limited component of reactions catalyzed by acetylcholinesterase. Biochemistry. 1986;25:125-130.

13. Massoulie J, Pezzementi L, Bon S, et al. Molecular and cellular biology of cholinesterases. Prog Neurobiol. 1993;41:31-91.

14. Darreh-Shori T, Forsberg A, Modiri N, et al. Differential levels of apolipoprotein E and butyrylcholinesterase show strong association with pathological signs of Alzheimer's disease in the brain in vivo. Neurobiol Aging. 2011;32(2320):e15-32

15. Greenblatt HM, Kryger G, Lewis T, Silman I, Sussman JL. Structure of acetylcholinesterase complexed with (-)-galanthamine at 2.3 A resolution. FEBS Lett. 1999;463:321-326.

16. Bar-On P, Millard CB, Harel M, et al. Kinetic and structural studies on the interaction of cholinesterases with the anti-Alzheimer drug rivastigmine. Biochemistry (Moscow). 2002;41:3555-3564.

17. Kawakami Y, Inoue A, Kawai T, Wakita M, Sugimoto H, Hopfinger AJ. The rationale for E2020 as a potent acetylcholinesterase inhibitor. Bioorg Med Chem. 1996; 4:1429-1446.

18. Kryger G, Silman I, Sussman JL. Structure of acetylcholinesterase complexed with E2020 (Aricept): implications for the design of new anti-Alzheimer drugs. Structure. 1999;7:297-307.

19. Mesulam M-M, Guillozet A, Shaw P, Levey A, Duysen E, Lockridge O. Acetylcholinesterase knockouts establish central cholinergic pathways and can use butyrylcholinesterase to hydrolyze acetylcholine. Neuroscience. 2002;110:627-639.

20. Mushtaq G, Greig NH, Khan JA, Kamal MA. Status of acetylcholinesterase and butyrylcholinesterase in Alzheimer's disease and type 2 diabetes mellitus. CNS Neurol Disord: Drug Targets. 2014;13:1432-1439.

21. Macdonald IR, Rockwood K, Martin E, Darvesh S. Cholinesterase inhibition in Alzheimer's disease: is specificity the answer? J Alzheimers Dis. 2014;42:379-384.

22. Karlsson D, Fallarero A, Brunhofer G, et al. Identification and characterization of diarylimidazoles as hybrid inhibitors of butyrylcholinesterase and amyloid beta fibril formation. Eur J Pharm Sci. 2012;45:169-183.

23. Kamal MA, Qu X, Yu Q-S, et al. Tetrahydrofurobenzofuran cymserine, a potent butyrylcholinesterase inhibitor and experimental Alzheimer drug candidate, enzyme kinetic analysis. J Neural Transm. 2008;115:889-898.

24. Rizzo S, Riviere C, Piazzi L, et al. Benzofuran-based hybrid compounds for the inhibition of cholinesterase activity, $\beta$ amyloid aggregation, and $\mathrm{A} \beta$ neurotoxicity. $J$ Med Chem. 2008;51:2883-2886.

25. Carolan CG, Dillon GP, Khan D, et al. Isosorbide-2-benzyl carbamate-5-salicylate, a peripheral anionic site binding subnanomolar selective butyrylcholinesterase inhibitor. J Med Chem. 2010;53:1190-1199.

26. Nicolet Y, Lockridge O, Masson P, Fontecilla-Camps JC, Nachon F. Crystal structure of human butyrylcholinesterase and of its complexes with substrate and products. $J$
Biol Chem. 2003;278:41141-41147.

27. Cheung J, Rudolph MJ, Burshteyn F, et al. Structures of human acetylcholinesterase in complex with pharmacologically important ligands. J Med Chem. 2012;55:10282-10286.

28. Dvir H, Silman I, Harel M, Rosenberry TL, Sussman JL. Acetylcholinesterase: from 3D structure to function. Chem Biol Interact. 2010;187:10-22.

29. Saxena A, Redman AM, Jiang X, Lockridge O, Doctor B. Differences in active site gorge dimensions of cholinesterases revealed by binding of inhibitors to human butyrylcholinesterase. Biochemistry. 1997;36:14642-14651.

30. Nachon F, Carletti E, Ronco C, et al. Crystal structures of human cholinesterases in complex with huprine $\mathrm{W}$ and tacrine: elements of specificity for anti-Alzheimer's drugs targeting acetyl- and butyryl-cholinesterase. Biochem J. 2013;453:393-399.

31. McGregor MJ, Pallai PV. Clustering of large databases of compounds: using the MDL "keys" as structural descriptors. J Chem Inf Comput Sci. 1997;37:443-448.

32. Lipinski CA, Lombardo F, Dominy BW, Feeney PJ. Experimental and computational approaches to estimate solubility and permeability in drug discovery and development settings. Adv Drug Deliv Rev. 1996;23:3-25.

33. Felder CE, Botti SA, Lifson S, Silman I, Sussman JL. External and internal electrostatic potentials of cholinesterase models. J Mol Graph Model. 1997;15:335-337.

34. Nolte HJ, Rosenberry TL, Neumann E. Effective charge on acetylcholinesterase active sites determined from the ionic strength dependence of association rate constants with cationic ligands. Biochemistry (Moscow). 1980;19:3705-3711.

35. Bourne Y, Radić Z, Sulzenbacher G, Kim E, Taylor P, Marchot P. Substrate and product trafficking through the active center gorge of acetylcholinesterase analyzed by crystallography and equilibrium binding. J Biol Chem. 2006;281:29256-29267.

36. Bartolucci C, Stojan J, Yu Q, Greig NH, Lamba D. Kinetics of torpedo californica acetylcholinesterase inhibition by bisnorcymserine and crystal structure of the complex with its leaving group. Biochem J. 2012;444:269-277.

37. O’Boyle NM, Banck M, James CA, Morley C, Vandermeersch T, Hutchison GR. open babel: an open chemical toolbox. J. Cheminformatics. 2011;3:33.

38. McGann M. FRED pose prediction and virtual screening accuracy. J Chem Inf Model 2011;51:578-596.

39. McGaughey GB, Sheridan RP, Bayly CI, et al. Comparison of topological, shape, and docking methods in virtual screening. J Chem Inf Model. 2007;47:1504-1519.

40. McGann MR, Almond HR, Nicholls A, Grant JA, Brown FK. Gaussian Docking Functions. Biopolymers. 2003;68:76-90.

41. Babu MN, Elumalai K, Srinivasan S, Eluri K, Elumalai M, Sivannan S. Synthesis and anticholinesterase activity of a novel series of acetazolamide condensed 1,4-dihydropyridines. Carbon Resour Convers. 2019;2:191-197.

42. Zafar AM, Iqbal A, Ashraf M, et al. Synthesis and in vitro cholinesterase inhibitory potential of dihydropyridine derivatives. Pakistan J Pharm Sci. 2019;32:1155-1162.

43. Darvesh S, Macdonald IR, Martin E. Selectivity of phenothiazine cholinesterase inhibitors for neurotransmitter systems. Bioorg Med Chem Lett. 2013;23:3822-3825.

44. Darvesh S, McDonald RS, Penwell A, et al. Structure-activity relationships for inhibition of human cholinesterases by alkyl amide phenothiazine derivatives. Bioorg Med Chem. 2005;13:211-222.

45. Qizilbash N, Whitehead A, Higgins J, et al. Cholinesterase inhibition for Alzheimer disease: a meta-analysis of the tacrine trials. J Am Med Assoc. 1998:280:1777-1782.

46. Irwin JJ, Sterling T, Mysinger MM, Bolstad ES, Coleman RG. ZINC: a free tool to discover chemistry for biology. J Chem Inf Model. 2012;52:1757-1768.

47. Wandhammer M, de Koning M, van Grol M, et al. A step toward the reactivation of aged cholinesterases-crystal structure of ligands binding to aged human butyrylcholinesterase. Chem Biol Interact. 2013;203:19-23.

48. Brus B, Kosak U, Turk S, et al. Discovery, biological evaluation, and crystal structure of a novel nanomolar selective butyrylcholinesterase inhibitor. J Med Chem. 2014;57:8167-8179.

49. Nachon F, Carletti E, Ronco C, et al. Crystal structures of human cholinesterases in complex with huprine W and tacrine: elements of specificity for anti-Alzheimer's drugs targeting acetyl-and butyryl-cholinesterase. Biochem J. 2013;453:393-399.

50. Knez D, Brus B, Coquelle N, Sosič I, Šink R, et al. Structure-based development of nitroxoline derivatives as potential multifunctional anti-Alzheimer agents. Bioorg Med Chem. 2015;23:4442-4452

51. Qi S, Peng D, Yang S, Xiao-Lei Z, Yang W, Guang-Fu Y. Syntheses of Coumarin-tacrine hybrids as dual-site acetylcholinesterase inhibitors and their activity against butylcholinesterase, $\mathrm{A} \beta$ aggregation, and $\beta$-secretase. Bioorg Med Chem. 2014:22:4784-4791.

52. Ellman G, Courtney K, Andres V, Featherstone R. Biochem Pharmacol. 1961;7:88-95

53. Joubert J, Foka GB, Repsold BP, Oliver DW, Kapp E, Malan SF. Synthesis and evaluation of 7-substituted coumarin derivatives as multimodal monoamine oxidase-B and cholinesterase inhibitors for the treatment of Alzheimer's disease. Eur J Med Chem. 2017;125:853-864.

54. Denya I, Malan SF, Enogieru AB, et al. Design, synthesis and evaluation of indole derivatives as multifunctional agents against Alzheimer's disease. Medchemcomm 2018;9:357-370

55. Zindo FT, Malan SF, Omoruyi SI, Enogieru AB, Ekpo OE, Joubert J. Design, synthesis and evaluation of pentacycloundecane and hexacycloundecane propargylamine derivatives as multifunctional neuroprotective agents. Eur $J$ Med Chem. 2019;163:83-94

56. Kapp E, Visser H, Sampson SL, et al. Versatility of 7-substituted Coumarin molecules as antimycobacterial agents, neuronal enzyme inhibitors and neuroprotective agents. Molecules. 2017;22:1644. 